\title{
Optimal Sorting Policies in Remanufacturing Systems: Application of Product Life-Cycle Data in Quality Grading and End-of-Use Recovery
}

\author{
Ardeshir Raihanian Mashhadi \\ ardeshir@buffalo.edu \\ Graduate Research Assistant \\ Mechanical and Aerospace Engineering \\ University at Buffalo, State University of New York \\ Buffalo, NY, USA \\ Sara Behdad \\ sarabehd@buffalo.edu \\ Assistant Professor \\ Mechanical and Aerospace Engineering \\ Industrial and Systems Engineering \\ University at Buffalo, State University of New York \\ Buffalo, NY, USA \\ Phone: 716-645-5914
}

\begin{abstract}
:
The quality of used products returned back to recovery facilities is often highly uncertain. Quality grading and sorting policies are immediate solutions that are used in remanufacturing systems to handle this source of variability in incoming products. The sorting policies offered in the literature so far are mainly based on external criteria such as market trends, corporate policies and assessment of the product's physical condition. In this study, we offer a new sorting method based on both product's internal factors such as future reusability of components, product identity data, and product health status as well as external factors such as market trends. The purpose of this paper is to improve decision making in remanufacturing operations by integrating the product life cycle information, particularly product usage phase data, into determining both optimal sorting policies and End-of-Life/End-of-Use (EoL/EoU) decisions. To achieve this, two related analyses are conducted: first, a reusability index is derived for each product unit based on the reusability of its components, product features, and the product usage phase information. Second, the reusability index is used as a quality measure to derive the optimal EoU decision for each product category. Clustering algorithms are employed to identify similar products that could go through the same recovery process. A data set of hard disk drive Self-Monitoring Analysis and Reporting Technology (S.M.A.R.T.) coupled with simulated data has been analyzed to illustrate the application of the model. The proposed framework helps decision makers include product identity data in the EoU recovery decision making under the quality heterogeneity.
\end{abstract}

\section{Keywords}

Product Recovery, Used Product Sorting, Clustering, Data-driven Remanufacturing, WEEE, Product Life Cycle Data 


\section{1- Introduction}

As the manufacturing industry is moving toward a more resource-constrained economy, the notion of resource security and sustainability of manufacturing systems requires more attention. One solution proposed to improve the sustainability of manufacturing systems is to adopt the concept of a circular economy in which new approaches are employed to keep the product value within the same manufacturing system through facilitating end-of-use recovery and remanufacturing operations [1]. However, remanufacturing operations suffer from many challenges including several sources of uncertainty in addition to the traditional uncertainties exist in the manufacturing frameworks [2]. For instance, unlike manufacturing systems, the condition of products acquired in remanufacturing facilities is highly variable. The quality level directly determines the buyback price, corresponding to the operational efforts required to remanufacture them. Therefore, quality grading of the incoming products in remanufacturing system is a critical challenge, since the high level of uncertainty and variability cripples the planning efforts in remanufacturing operations.

In order to develop a proper quality grading system in remanufacturing facilities, two general steps are needed. First, an accurate measure should be defined to assess the quality of incoming products and second the measure should be employed to sort and categorize the products with the aim of maximizing the profit out of EoU recovery decisions.

Currently, two common quality assessment approaches are used in the industry. The first approach is based on the physical inspection of returned products. This approach may require full disassembly of the product, which is a labor-intensive process and is not cost efficient [3]. The second approach, which is more common in the consumer electronics industry, is the nominal quality grading based on the product appearance and basic functionalities [4]. The latter lacks the proper accuracy as assessing the quality based on appearances, market trends, corporate policies and basic features may not reveal a comprehensive condition of the product.

Overall, quality grading and product sorting policies have been the point of attention in the closed-loop supply chain literature [5-7]. There is a consensus in the literature that suggests proper quality grading prior to remanufacturing decisions ultimately improves the profit $[7,8]$. However, these studies are founded upon similar assumptions regarding a priori knowledge of the statistical distributions of the product quality or error free inspections. Therefore, new approaches are still needed to overcome these assumptions. To fill this gap and further to use the opportunities available through improvements of data science and advancements in the sensing technologies during recent years, data-driven approaches have been proposed to utilize product life cycle information for reusability and quality assessment purposes [911]. The main idea of such studies is to integrate one or several sensors in the product to record data over its usage cycle. Then, these data are used to determine the real-time product quality level or predict its failure. The same source of data can be used to identify the product's quality grade or its reusability potential for EoU decision making [12].

Although the idea of using product sensor data for EoU recovery decisions has already been discussed in the literature, no effort has been made to implement this idea. Research is still in the early stage in this field. Therefore, there is a need for a comprehensive quality assessment model that allows using product life cycle data with the aim of simultaneous consideration of both internal and external product information such as product features and conditions as well as market trends in sorting decisions. In addition, the proposed models should be able to handle product lifecycle data, independent of how and 
where data are collected, sensors data or other collection methods. Furthermore, more research should be conducted regarding the impact of product life cycle data on the EoU decision making process and the way that product identity data should be employed. In many cases, even if the product identity data for each unit of product are available, it is not practical to tailor remanufacturing operations for each individual product, unless some sort of clustering and grouping is used to minimize the remanufacturing operational costs.

The current paper contributes to the literature by addressing the above mentioned gaps. First, we propose a method that uses product life cycle data (e.g. product identity data) to determine product quality conditions and quantify the future reusability. Second, we show how products with similar quality levels can be clustered to facilitate decision making in remanufacturing operations, and finally we illustrate how best EOU decisions will be made for each product cluster. A product reusability index has been proposed based on the product critical component features and life cycle information. The reusability index defines the quality level of the product. Clustering algorithms are used to sort and group the products based on their quality level and an optimization problem is defined to determine the best recovery operation for each cluster. The application of the proposed method has been shown in a case study of computer hard drives.

The rest of this paper is organized as follow: Section 2 provides a review of literature on the quality grading and the integration of product life cycle information in EoU recovery decisions. Section 3 discusses the proposed method including the quality assessment step, sorting policy and the EoU decision model. Section 4 provides a case study of personal computers. Section 5 presents the sensitivity analysis on the model results and finally, Section 6 concludes the paper.

\section{2- Background: Quality Grading Models and Sorting Policies}

Since the uncertain condition of returned products significantly affects the testing, disassembly and EoU recovery decisions, addressing the uncertainty in the quality of returns prior to remanufacturing is becoming critical. It has been shown that as the product sorting and acquisition process improves, the profitability of recovery operations improves as well [13]. This section strives to provide a review of studies that address the issue of uncertain quality returns in EoU recovery literature.

\section{1- Different Approaches in Modeling Uncertainties in the Quality of Returns}

Generally, two types of modeling approaches have been employed in the literature to address the uncertainty in the quality of returned products [14]. The first category of studies considers a fraction of remanufacturable products in each batch of returns. The second group of studies represents the quality of returns by using a continuous random variable between zero and one with known probability distribution.

To mention a few studies, Pishvaee et al. [15] assumed that an inspection stage separates the products into two groups: recoverable (high quality) and unrecoverable (low quality) products. Fathi et al. [16] considered an inspection based quality assessment system where the quality of a product is defined based on the expected processing time required for remanufacturing it. Therefore, products are separated into two groups; those that have a processing time less than a threshold value and those that have a processing time greater than the threshold. The latter will be rejected for remanufacturing.

Zikopoulos and Tagaras [5] also assumed that the quality of the product is determined after inspection and defined nominal quality grades to investigate the impact of uncertainty in the quality of returns on the profit of the operations. Similarly, Denizel et al. [6] considered nominal quality grades based on inspection for a multi-period remanufacturing system. Ferguson et al. [8] used a real number varying 
between zero and one to model the quality of the product. Galberth and Blackburn [17] and Panagiotidou et al. [14] used known statistical distributions to formulate the uncertainty in the quality of returns. Aligned with the previous studies, Subulan et al. [18] developed a stochastic mixed integer linear programing model for lead/acid battery closed-loop supply chain. They considered an uncertain quality level for batteries where the ratio for inappropriate condition of the batteries is stochastic. Francie et al. [19] studied a hybrid manufacturing/remanufacturing system for printer cartridge industry where products are separated into two categories based on their quality levels.

\section{2- Integration of Product Life Cycle Data into End-of-Life Recovery Decisions}

Regardless of the mathematical approach to model the uncertainty in the quality of the products, a more practical challenge is to define the measure to represent the quality level. Although using nominal quality grading approaches based on the product appearances and basic features is more common in the industry (readers may refer to [4,20] as examples of quality assessment in web-based trade-in programs), utilization of product life cycle information to represent its quality level seems more promising, as it brings more accuracy and flexibility into recovery operations.

The idea of using life cycle sensor data for quantifying reuse potential in electronic products was first introduced by Scheidt and Zong [12]. They emphasized on the advantages of having a modular design and introduced an "identification unit" for each module to record the life cycle data. In their model, the data were accessible using a "green port". The idea was followed by Klausner et al. [21]. They integrated a sensor-based circuit called "Electronic Data Log" into products in order to record the product degradation data over its life cycle and studied the further usage of the data in product recovery systems. In another study, Klausner et al. [22] also addressed the issue of misclassification using the electronic data $\log$ module and discussed that the return rate of the products plays a pivotal role in the overall efficiency of such systems. Simon et al. [23] studied the life cycle data acquisition in a washing machine case study in the UK. They used static data from the manufacturer and dynamic life cycle data gathered from employed sensors in the product to read the measurements of environmental parameters. The information was used to reduce the cost while satisfying the demand for service parts.

Teunter and Flapper [24] also investigated core acquisition and remanufacturing policy under quality and demand uncertainty. They incorporated known statistical distributions to model the probability that a core belongs to a certain quality level. Jin et al. [25] proposed a Markov decision process model for the case in which the probability that each module is remanufacturable and the probability that each remanufacturable module belongs to a certain quality level are constant. They showed that the optimal policy presents significant benefits compared to the exhaustive reassembly policy. Ondemir and Gupta [26] used Radio Frequency Identification (RFID) and sensor data to capture the remaining useful life of products as a measure of quality in EoL decision making. Jun et al. [27] also developed a framework to use RFID data in product life cycle management applications.

In spite of the fact that the idea of installing sensors into the products to store the components' degradation data for future product recovery decisions was proposed around two decades ago [12], there are still major challenges available to implement such systems. Zikopoulos and Tagaras [28] claimed that using data recorded in the products as a substitute for $100 \%$ inspection may be prone to misclassification and analyzed the attractiveness of a simple sorting procedure with limited accuracy prior to remanufacturing. They considered two classes of quality grades, remanufacturable and not remanufacturable, and concluded that the performance of such systems depends on the accuracy of the sorting process and the expected yield of the returned products. In another study, Van Wassenhove and Zikopoulos [29] considered more variability in the quality grades and investigated the impact of 
misclassifications in the quality grading of to-be-remanufactured cell phones, particularly quality overestimation, on the profit of a remanufacturing firm. They proposed that appropriate sorting methods can be implemented in order to minimize the probability of misclassification. Parlikad and McFarlane [30] have extended the concept of Baye's factor, which can be typically used as a Bayesian alternative for hypothesis testing problem, to a measure for the strength of evidence provided by a piece of information and investigated the value of information about the product quality in recovery decisions. They showed that the penalty for misclassification of products, the level of uncertainty about the quality of products and the strength of information determine whether the information is valuable for recovery decisions or not. On the other hand, Reveliotis [31] noted that any a priori assumption regarding the full availability of information about statistical distributions of cost data or quality classes in the disassembly process are unrealistic and therefore used learning methods to find approximates for optimal disassembly planning problem.

Although installing sensors in the products, which are primarily designed for recording degradation data over product life cycle mainly for the purpose of maintenance, looks promising from an EoU recovery perspective, more studies are needed to show how these data can be used to facilitate informed decision making at EoU stage. Data-driven methods have been used with the aim of engineering systems health prognosis and remaining useful life estimation (e.g., [32,33]). Therefore, data-driven recovery methods may be extended to reveal the novel applications of the sensor data. For instance, data collected during product lifecycle show that different groups of consumers (i.e., household vs. commercial) reveal distinct usage behaviors (i.e., usage hours) even for a similar product [34]. This segregation may enable the use of product life cycle information in sorting and quality grading.

On the other hand, even upon availability of the product life cycle information at the EoU recovery point, the impact of such information on the profitability of the recovery operations should be investigated further. As mentioned before, data-driven quality grading methods are prone to misclassification and their advantage is in the pace of the process and the ability to fast-sorting the products and components prior to remanufacturing. However, decision making on a per product basis may be redundant and impractical for remanufacturing firms, as it increases the complexity of the decision making and may decrease the resource allocation and utilization.

This paper strives to present a generic yet comprehensive model to capture the product reusability index based on the life cycle information. We have also used clustering techniques to group similar products with respect to their quality grade in order to ease the decision making process. The method is based on the data retrieved from the product usage phase. The proposed framework is generic and can be applied to any sort of data that indicate the product quality or reusability level.

\section{3- Method: A Data-driven Approach for Product Quality Grading, Sorting and End-of-Life Decision Making}

The proposed method begins with assessing the quality grade of returned products. In this method, a reusability index will be calculated as a measure for the quality of products and their components. The overall procedure for the value recovery process is as follow: 1) First the reusability index of the components is assessed based on the available usage information of that component and other relevant features of the component that may indicate its level of reusability. 2) Second, the reusability level of the product is determined based on the reusability indices of its components. 3) After the reusability indices of the products are calculated, the products are clustered and then sorted based on the similarities between their quality levels (i.e. reusability indices) of their components. 4) This information is then passed on to an optimization problem in the EoU decision making in order to find the best EoU fate for each cluster 
using its quality level, corresponding recovery and inventory costs and the demand. Figure 1 illustrates the overall process of sorting, clustering and EOU decision making. The details of each step are discussed in the rest of this section.

We assume that any used product $(j \in\{1,2, \ldots, N\})$ contains several critical components $(i \in$ $\{1,2, \ldots, n c c\})$. These components are those that directly control the functionality of the product such that they do not just add features, but the product loses its basic functionality without them. Another pivotal interpretation regarding the critical components is that these components can represent the parts that are more likely to fail. We define vector $f_{i j}=\left(f_{i j 1}, \ldots, f_{i j P}\right)$ such that $f_{i j}$ represents the $P$ important features of component $i$ that are used as criteria to define the reusability level of component $i$ in product $j$. The reusability index for component $\mathrm{i}$ in product $\mathrm{j}$ is defined as follow:

$$
R_{i j}=\sum_{p=1}^{p=P} \beta_{i p} f_{i j p}
$$

The coefficients $\beta_{i p}$ should be defined based on the remanufacturing firm's knowledge about the market trends and the importance of component features. These coefficients are flexible and can be changed regarding the market conditions. For example, for a computer hard drive the important features that affect the EOL decision can be, inter alia, size, age, actual usage life and current price of the hard drive in the market. Therefore, in the case that technological obsolescence plays a pivotal role, age should have a larger coefficient, but when the demand for larger hard drive capacity dominates the market, a larger coefficient should be assigned to size. We use the reusability index as a measure of the component quality and hence the product quality level. Defining a multidimensional quality measure in comparison with a single measure like remaining useful life, has the advantage of flexibility for the firm. For example, an old product which was barely used may indicate a high remaining useful life. However, due to the fast paced technology obsolescence in the case of consumer electronics, such a product may not be considered as high quality.

The quality of each product is defined based on the reusability index of the product and is therefore derived from the reusability indices of its critical components. Since the critical components control the functionality of the product, we assume that the reusability index of the product equals to the minimum of the reusability indices of its components. Hence,

$$
R_{j}=\left\{\min R_{i j} \mid i \in\{1, \ldots, n c c\}\right\}
$$

Remanufacturing, refurbishing and material recovery (recycling) are assumed to be three available recovery operations. The sequence of the activities is as follow. First, the reusability indices of its critical components are assessed. Then the reusability indices are used to represent the quality level of the product. Products with larger reusability indices have higher quality grades and those that have smaller reusability indices have lower quality grades, respectively. After the reusability estimation, products with similar quality condition will be grouped into the same clusters. A k-means clustering algorithm has been used to group the products with quality resemblance. We used k-means due to its fast computational speed and the flexibility of implementation. However, one limitation of k-means clustering is the required prior knowledge about the number of clusters. Therefore, we first determine the optimum number of the clusters based on the data and then feed the clustering results to the optimization problem. The recovery decision should be made after the products are categorized.

Refurbishment includes operations like software update and cleaning that lets the product to be used for its current reusability level. However, remanufacturing involves replacing the critical components that 
have low reusability levels with new components, which means resetting their usage time to zero. Therefore, the total reusability index of the products will increase to a certain desired level. If neither of the two recovery options is profitable, the product will be recycled for material harvesting. We assume that recycling has a constant profit.

Several studies have previously highlighted the impact of uncertain demands on the profitability of recovery operations $[33,34]$ Thus, the demand is assumed to be known to the firm. In addition, specific demand is considered for each reusability level. Therefore, the demand is categorized according to the product clusters calculated in the model. The recovery decision made for each cluster will be applied to all the products within that cluster. However, the reusability levels of the centroids of the clusters are used in the decision process to represent the quality level of that cluster. Since, in the remanufacturing process critical components are replaced and therefore the reusability level of the products is enhanced, the remanufactured products may belong to a higher quality grade cluster after the remanufacturing. Therefore, the refurbished and remanufactured products are re-categorized in the available clusters so that remanufactured products with updated quality levels are grouped in their new clusters. Excess products with respect to the demand in each cluster will be sent to the inventory. Thus, we aim to find the best recovery strategy for the clusters such that the total profit is maximized. The optimization problem for EOU recovery decision making is extended from previous work of the authors [35].

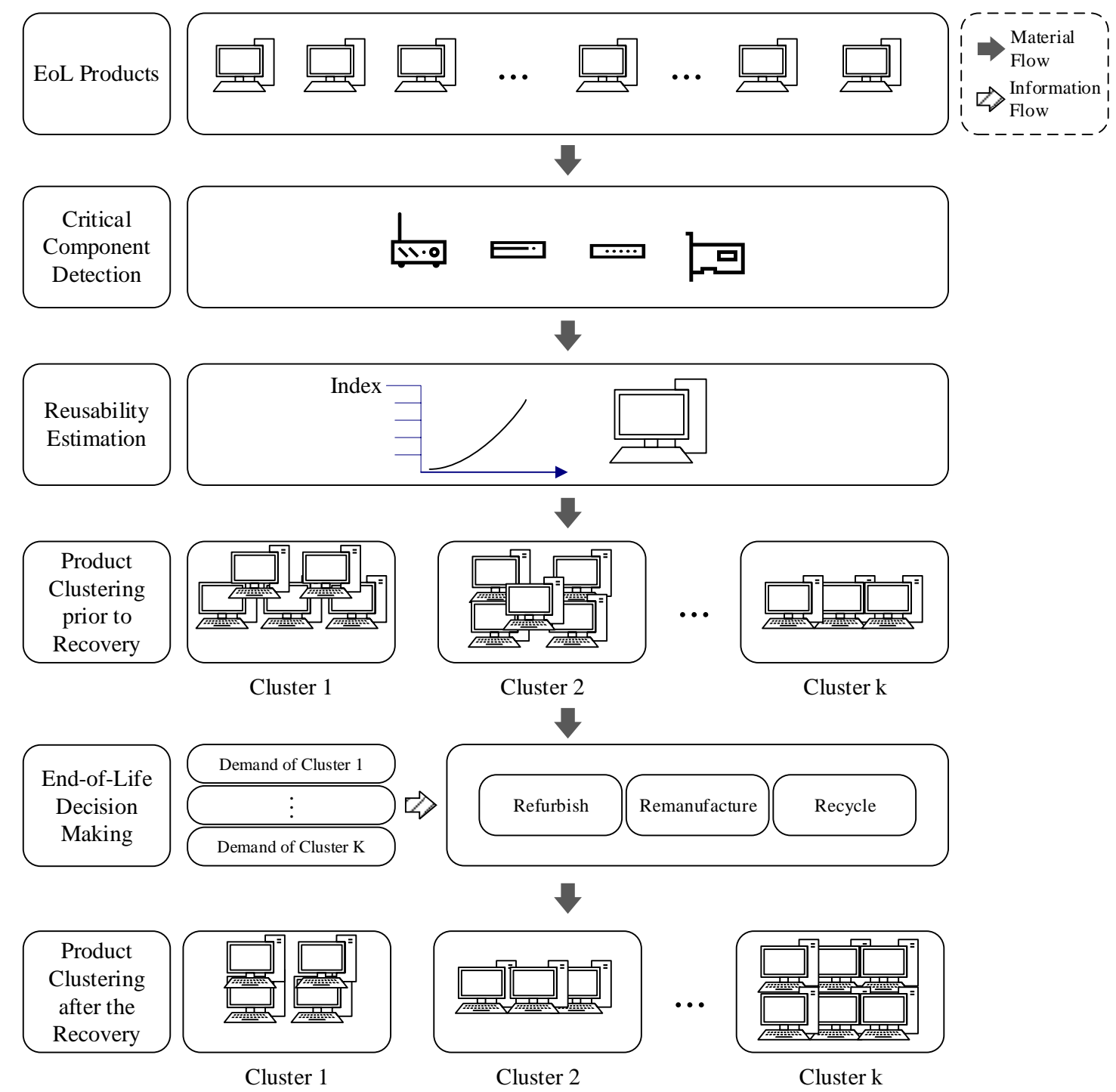


Figure 1- The proposed model for the EOU recovery process.

Table 1 - Variables of the model (extended from [35]).

\begin{tabular}{|c|c|c|c|c|}
\hline \multicolumn{2}{|c|}{ Variables } & Definition & Parameters & Definition \\
\hline \multirow{11}{*}{ 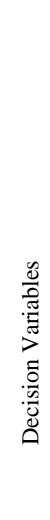 } & $n b_{k}$ & Number of products in cluster k before recovery & $\mathrm{N}$ & Number of products \\
\hline & $n a_{k}$ & Number of products in cluster $\mathrm{k}$ after recovery & ncc & Number of critical components \\
\hline & $\mathrm{nr}$ & Number of products sent for recycling & $\mathrm{K}$ & Number of clusters \\
\hline & $\mathrm{ns}_{\mathrm{k}}$ & Number of sold products in cluster $\mathrm{k}$ & $\mathrm{RPC}_{\mathrm{i}}$ & Replacing cost for component $\mathrm{i}$ \\
\hline & $n i_{k}$ & Number of products sent to inventory for cluster $\mathrm{k}$ & RFC & Refurbishing cost per product \\
\hline & TC & Total cost of the process & $\mathrm{HC}$ & Holding cost per item \\
\hline & $\gamma_{k}$ & 1 if cluster $\mathrm{k}$ is refurbished, zero otherwise & $D_{k}$ & Demand for cluster $\mathrm{k}$ \\
\hline & $\varphi_{k}$ & 1 if cluster $\mathrm{k}$ is remanufactured, zero otherwise & $\mathrm{P}_{\mathrm{k}}$ & Selling price of cluster $\mathrm{k}$ \\
\hline & $\delta_{k}$ & 1 if cluster $\mathrm{k}$ is recycled, zero otherwise & PR & $\begin{array}{l}\text { Profit per unit product } \\
\text { recycling }\end{array}$ \\
\hline & $\varphi_{k i}$ & $\begin{array}{l}1 \text { if component } \mathrm{i} \text { of products in cluster } \mathrm{k} \text { is } \\
\text { remanufactured, zero otherwise }\end{array}$ & $R_{i j}$ & $\begin{array}{l}\text { Reusability index of component } \\
i \text { of product } j\end{array}$ \\
\hline & & & $R_{j}$ & Reusability index of product $\mathrm{j}$ \\
\hline
\end{tabular}

The objective is to find the best recovery option for each product cluster such that the total profit of the recovery operations is maximized. The decision variables of the EoL decision making problem are $\gamma_{k}, \varphi_{k}$ and $\delta_{k}$. Therefore the objective is as follows:

$$
\text { Max : Profit }=n r P R+\left(\sum_{i=1}^{K} n s_{k} P_{k}\right)-T C
$$

The first term of the objective function refers to the profit obtained by the recycling and the second term corresponds to the revenue of the recovered (refurbished and remanufactured) products sold to the market. The last term is the total cost of the process which contains the costs of remanufacturing, refurbishing and inventory.

In addition to the trivial sign constraints that entail all the variables to be positive, the following constraints are considered:

Any product and hence any product cluster that enters the facility will be refurbished, remanufactured or recycled. Therefore:

$$
\gamma_{k}+\varphi_{k}+\delta_{k}=1 \quad \forall k \in\{1,2, \ldots K\}
$$

Remanufacturing includes any replacement of the critical components:

$$
\text { if } \varphi_{k i}=1 \rightarrow \varphi_{k}=1 \forall k \in\{1,2, \ldots, K\}, \forall i \in\{1,2, \ldots, n c c\}
$$

The above mentioned conditional constraint can be written as the following:

$$
\varphi_{k} \geq \sum_{i=1}^{n c c} \varphi_{k i}
$$




$$
\varphi_{k} \leq A \sum_{i=1}^{n c c} \varphi_{k i}
$$

Where, A is any positive large number.

The total cost of the operations includes the costs of all the recovery options. The first term corresponds to the cost of remanufacturing, second term corresponds to the cost of refurbishing and the last term represents the inventory cost:

$$
T C=\sum_{k=1}^{K} \sum_{i=1}^{M} \varphi_{k i} n b_{k} R P C_{i}+\sum_{k=1}^{K} \gamma_{k} n b_{k} R F C+\sum_{k=1}^{K} n i_{k} H C
$$

Number of the products prior and after the recovery operations should be similar:

$$
\sum_{k=1}^{K} n b_{k}=\sum_{k=1}^{K} n a_{k}+\sum_{k=1}^{K} n i_{k}
$$

The maximum number of products that can be sold in each cluster equals the demand for that cluster and the rest of the products should be sent to the inventory:

$$
\begin{array}{ll}
\min \left(D_{k}, n a_{k}\right)=n s_{k} & \forall k \in\{1,2, \ldots, k\} \\
n a_{k}-n s_{k}=n i_{k} & \forall k \in\{1,2, \ldots, k\}
\end{array}
$$

\section{4- Case Study: Personal Computers}

This study is motivated by a real case of a remanufacturing facility which accepts donations of used consumer electronics. The remanufacturer is located in Chicago, Illinois, USA. The data are from more than 44000 collected items during 2011-2013 turned back by consumers with no charge or incentive. The received products should be tested and sorted so that the best recovery decision is made for each of them. The majority of the products received by the firm are personal computers and the processes of testing and sorting them are time consuming and challenging. We strive to develop a model to facilitate the quality assessment and sorting of the products. The objective is to develop a framework for fast sorting of the personal computers, even with some compromise in the accuracy.

The firm records the return date for each computer and retrieves the Self-Monitoring Analysis and Reporting Technology (S.M.A.R.T.) data of the hard drives. The S.M.A.R.T data are easily accessible using third party free software packages or the Command Prompt in Windows. The S.M.A.R.T data report a variety of information about the design and technology characteristics of the hard drive such as manufacturing date, model, brand, capacity and the firmware version and usage information such as the power-on time. 
After preprocessing the data and dealing with the missing values and inaccurate date reports, a data set was generated containing the data of more than 600 hard drives of a certain brand. The following variables were derived for analysis:

Power_on_years: represents the total duration that the hard drive was powered on and therefore somehow indicates the actual usage time of the product. A study by Backblaze [36], a data backup company, suggests that the expected median hard drive life is around 6 years. Therefore, we assume that the expected life span of hard drives in the data set is 6 years. This assumption was used to estimate the remaining useful life (RUL) of the hard drives as a criterion for EoU decision making. The RUL estimation can be done by subtraction of the actual usage age of the device from the expected life of the device [37,38]. The RUL is a determinant of the quality of the product such that lower RUL indicates a higher technical obsolescence level and suggests that the product/component is more prone to failure.

Age: is calculated based on the manufacturing date of the device and the date that the device is donated to the remanufacturing facility. Age also is a good determinant of the reusability or quality level of the drive as it is an indicator of the technological obsolescence. For example, a 15-year old hard drive may be quite technologically obsolete with no potential demand in the market. Therefore, even with a high RUL, a very old device cannot have a high quality level. This is a reasonable assumption, particularly in the case of consumer electronics with high rates of obsolescence [39].

Device_capacity: the third criterion considered to calculate the reusability index of the hard drive is the hard drive size. Similar to the age, hard drive size is a measure for technological obsolescence while it also shows how reusable the drive is in the current market. For instance, a hard drive that was barely used indicates a high RUL, but with a very low size capacity may not be sufficiently valuable to refurbish.

After preprocessing the data, 523 hard drives and the corresponding feature values were available to analyze. Table 2 represents the descriptive statistics and Figure 2 illustrates the frequency and distributions of the features.

Table 2 - Descriptive statistics of the hard drive features

\begin{tabular}{llll}
\hline & Power on years & Age (Years) & Capacity $(\mathrm{Mb})$ \\
\hline Min & 0.000228 & 1 & 16844 \\
Median & 1.277626 & 6 & 76319 \\
Mean & 1.562202 & 5.6 & 103488 \\
Max & 3.987329 & 11 & 476940 \\
\hline
\end{tabular}



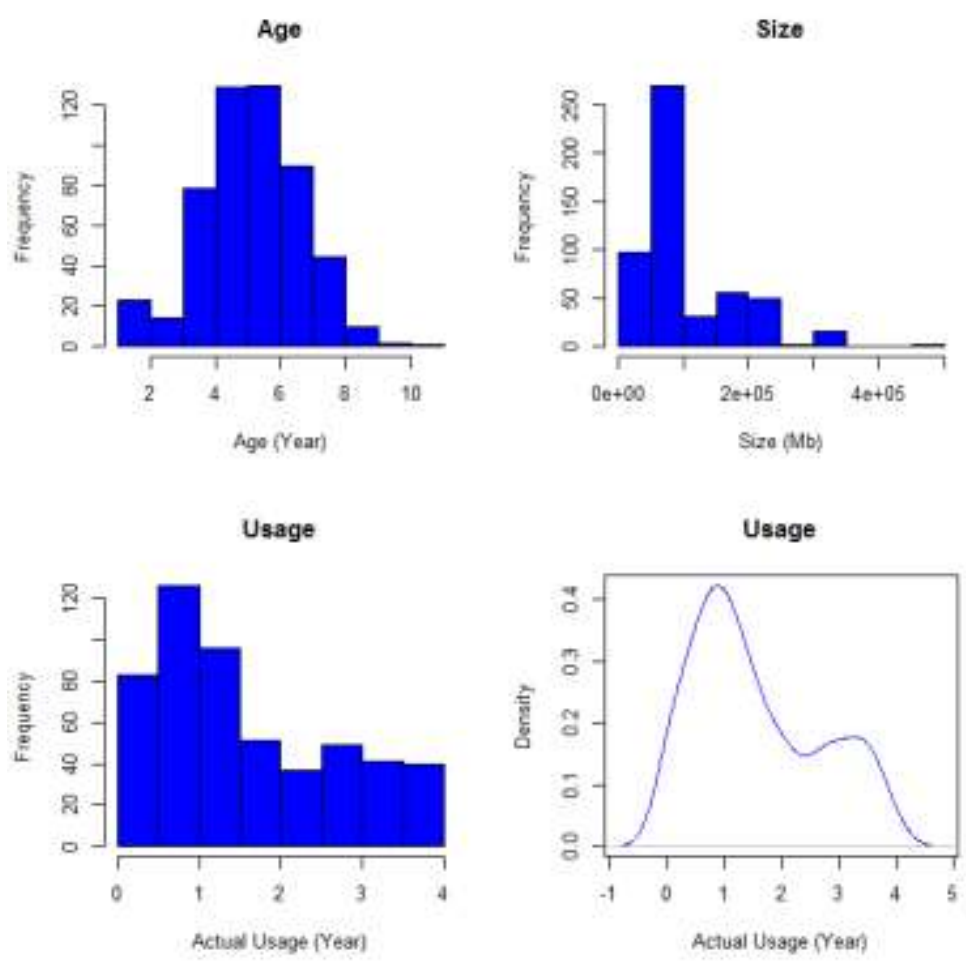

Figure 2 - Distribution of hard drive features

Although the power_on_years values were retrieved from the S.M.A.R.T data of the hard drives, it actually indicates the usage time of other components in the computer as well. Unless a component is replaced prior to the time that the product enters the remanufacturing facility, the powered on time of that component is similar to the hard drive. Therefore, the RUL of different components with different expected lives can be derived from the same data.

Since only hard drive data were available to us, in order to show the application of the model the RUL of another arbitrary component with an expected life of 4 years has been considered. To eliminate the effect of multicollinearity between the features, two other features for the second component have been simulated using truncated normal distributions. In other words, it is assumed that the products have two critical components, each of which has a feature vector with three features. Table 3 summarizes the input data used in the model and the corresponding coefficients to calculate the reusability index.

Table 3 - Summary of the input data

\begin{tabular}{ccccccc}
\hline & Feature 1 & Source & Feature 2 & Source & Feature 3 & Source \\
\hline $\begin{array}{c}\text { Component 1 } \\
(\mathrm{HDD})\end{array}$ & $\begin{array}{c}\text { Capacity } \\
(\mathrm{Mb})\end{array}$ & Dataset & Age & Dataset & RUL(Years) & Dataset \\
Component 2 & $\mathrm{X}$ & Simulated & $($ Years $)$ & Simulated & RUL(Years) & Dataset \\
\hline \multirow{2}{*}{ Coefficients } & $\beta_{11}$ & 0.5 & $\beta_{12}$ & 0.4 & $\beta_{13}$ & 0.1 \\
& $\beta_{21}$ & 0.4 & $\beta_{12}$ & 0.4 & $\beta_{13}$ & 0.2 \\
\hline
\end{tabular}


After calculation of the reusability index for each of the components, the model can be applied. In order to run the model, the optimum number of clusters should be found. The algorithm implemented here is kmeans clustering, which tends to divide the points into k clusters such that the within-cluster sum of squares is minimized [40]. In order to find the similarities of the points, Euclidian distance has been utilized. Two different methods have been tested for selecting the optimum number of clusters. First, Silhouette analysis that indicates which points are lied well within their clusters and which ones are in between clusters [41]. The average silhouette width is the measure that shows the goodness of clustering. The second measure is the gap statistics, which compares the change in within-cluster dispersion with a reference distribution [42]. Figure 3 illustrates the summary of the silhouette analysis on the number of clusters. Silhouette analysis has been done on the results of k-means clustering and as can be seen, the average silhouette width for $\mathrm{k}=2,3,4$ and 5 are $0.4,0.37,0.33$ and 0.32 respectively. Therefore, it suggests that the data points will lie within two clusters better and hence, the optimum number of clusters should be two.
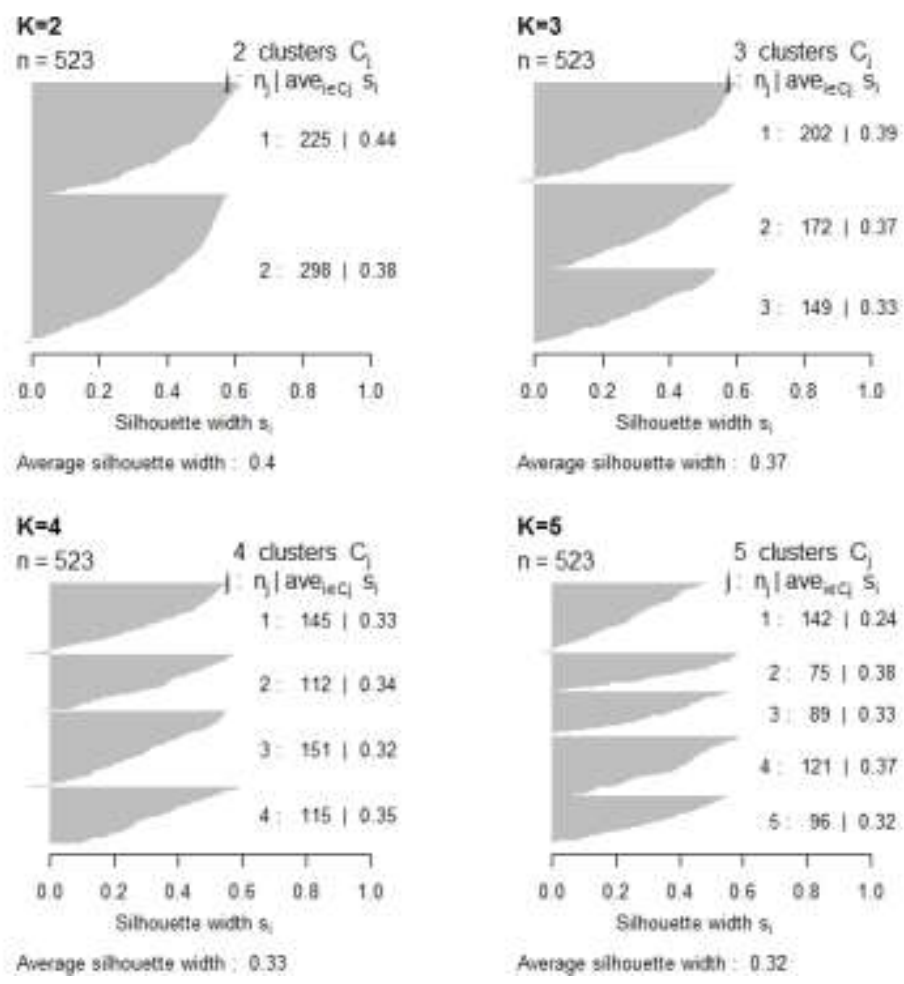

Figure 3 - summary of the silhouette analysis for $\mathrm{k}=2,3,4$ and 5 .

Figure 4 illustrates the results of the gap statistics test to find the optimum number of the clusters. The analysis has been carried out for $k \in\{1,2 \ldots 10\}$. The first local optima occurs when $\mathrm{k}=2$. Therefore, the results of the gap statistics measurements also suggest that the optimal number of clusters should be two. 


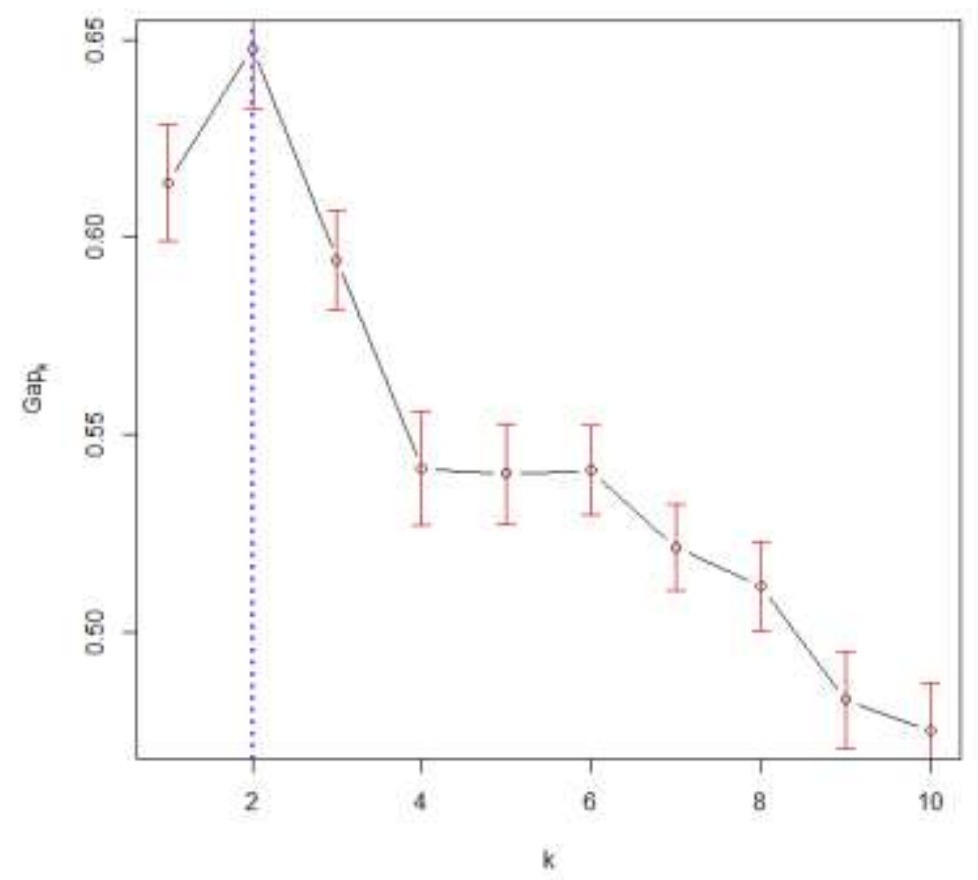

Figure 4 - Gap statistics for k-means clustering when $k \in\{1,2 \ldots 10\}$. The blue dotted line indicates the optimal number of clusters, $\mathrm{k}$.

After determining the optimum number of clusters, the optimization problem can be solved. Table 4 illustrates the optimization parameters. The optimization problem was solved using OptQuest Solver engine. It converges after 500 iterations to profit $=\$ 22,400$. The optimal decision found to be $\gamma_{2}=$ $\varphi_{1}=\varphi_{11}=1$ and the rest of the decision variables set to be zero. This means that the products in Clusters 2 should be refurbished and Component 1 in Cluster 1 products should be replaced. Figure 5 illustrates the detailed solution of the optimization problem.

Table 3 - The input parameters of the optimization problem

\begin{tabular}{lclc}
\hline Parameters & Initial Value & Parameter & Initial Value \\
\hline $\mathrm{N}$ & 523 & $\mathrm{HC}$ & $\$ 10$ \\
$\mathrm{ncc}$ & 2 & $\mathrm{D}_{1}$ & 150 \\
$\mathrm{~K}$ & 2 & $\mathrm{D}_{2}$ & 350 \\
$\mathrm{RPC}_{1}$ & $\$ 30$ & $\mathrm{P}_{1}$ & $\$ 50$ \\
$\mathrm{RPC}_{2}$ & $\$ 50$ & $\mathrm{P}_{2}$ & $\$ 80$ \\
$\mathrm{RFC}$ & $\$ 20$ & $\mathrm{PR}$ & $\$ 10$ \\
\hline
\end{tabular}




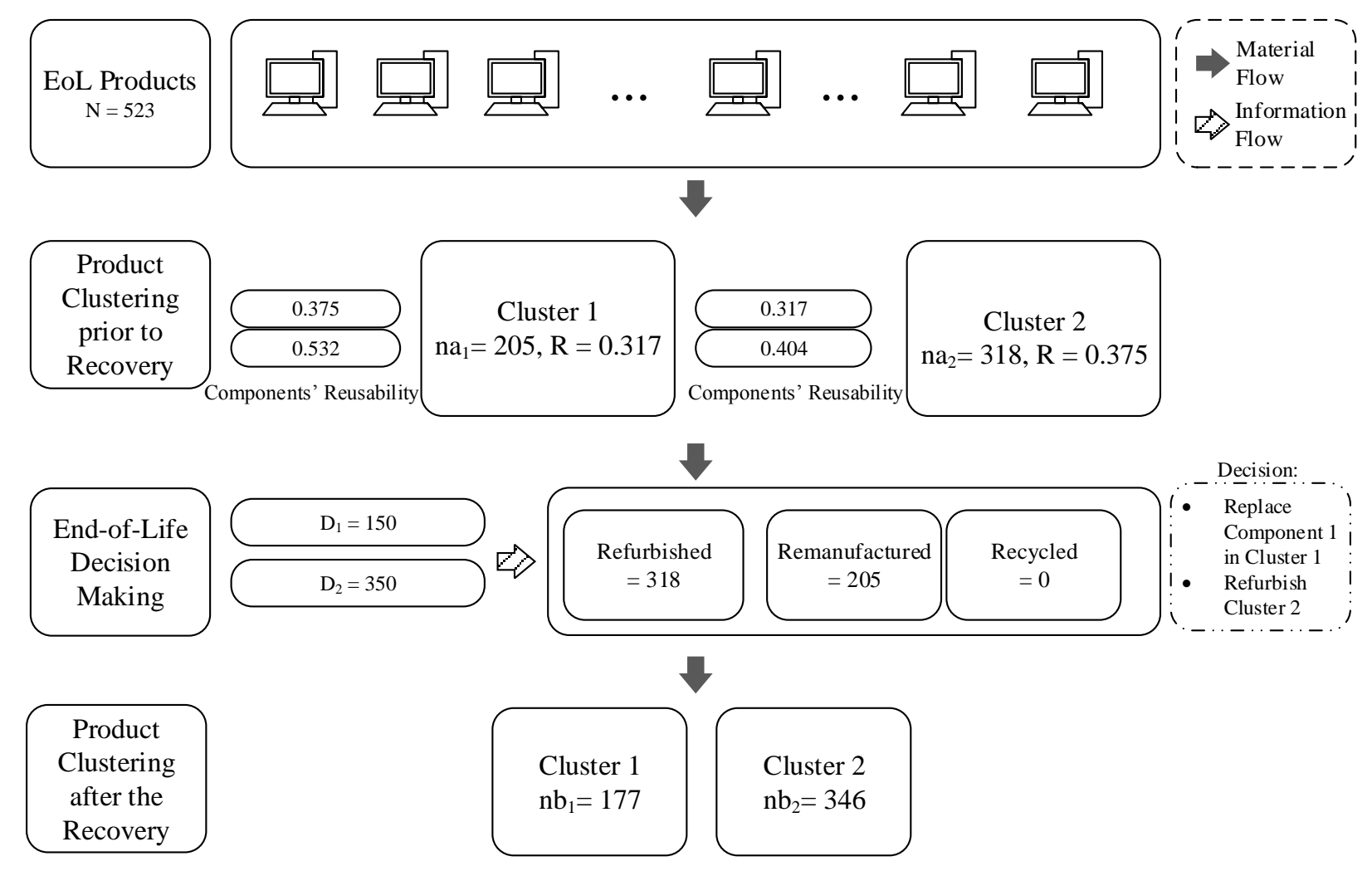

Figure 5 - The detailed solution of the optimization problem

\section{5- Sensitivity Analysis: Impact of Demand on Optimal Recovery Strategies}

A sensitivity analysis has been carried out in order to study the dependencies of the model to the input parameters and their significance. Table 4 summarizes the results of the sensitivity analysis. As we assumed that the demand is known to the firm, the analysis has been done to show the effect of different demand scenarios. Three regions have been considered to analyze the cases where the total demand is less than, equal to and greater than the number of input products. The regions are separated via shades Table 4. In each region four cases have been considered for different situations of the relative demand of the two clusters such as each case number refers to a specific setup for the demands of Clusters 1 and 2 . Two of the cases correspond to the instances that the demand for one of the clusters is extremely higher than the demand for the other cluster, while the other two cases represent the situation where the demands of the two clusters are comparable but one of which is relatively higher. As can be seen, in the first region, where the total demand is less than the number of products, no remanufacturing operations have been selected as the optimal strategy. In addition, the profit decreases with the decrease in the demand of the products in the second cluster which have higher values. In all four cases, the optimum strategy is to refurbish the products of the cluster that has a higher demand and recycle the other one. This is a reasonable strategy due to the fact that since the products are under-demanded remanufacturing them or holding them will result in high inventory costs. 
The second region corresponds to the case that the total demand equals the number of input products. As the price of the products in cluster two is higher, the final profit is significantly dependent on the number of Cluster 2 products that can be sold. In the first case where the only available demand is for Cluster 2, the optimum strategy is to refurbish Cluster 2 and remanufacture Cluster 1 in order to achieve as much as Cluster 2 quality products. In all the other cases, where more demand is available for Cluster 1, the optimum strategy is to maintain Cluster 1 by refurbishing its products and recycle or refurbish Cluster 2 based on the demand.

Table 4 - The results of the sensitivity analysis on the impact of demand on optimal EOU recovery decisions

\begin{tabular}{|c|c|c|c|c|c|c|c|c|c|c|c|c|c|c|c|}
\hline & \multirow[b]{2}{*}{ Case \# } & \multicolumn{3}{|c|}{ Demand } & \multicolumn{2}{|c|}{ Refurbish } & \multicolumn{6}{|c|}{ Remanufacture } & \multicolumn{2}{|c|}{ Recycle } & \multirow[b]{2}{*}{ profit } \\
\hline & & $D_{1}$ & $\mathrm{D}_{2}$ & total & $\gamma_{1}$ & $\gamma_{2}$ & $\varphi_{11}$ & $\varphi_{12}$ & $\varphi_{21}$ & $\varphi_{22}$ & $\varphi_{1}$ & $\varphi_{2}$ & $\delta_{1}$ & $\delta_{2}$ & \\
\hline \multirow{4}{*}{ 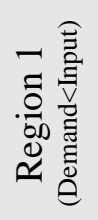 } & 1 & 0 & 300 & 300 & 0 & 1 & 0 & 0 & 0 & 0 & 0 & 0 & 1 & 0 & 19510 \\
\hline & 2 & 100 & 200 & 300 & 0 & 1 & 0 & 0 & 0 & 0 & 0 & 0 & 1 & 0 & 10510 \\
\hline & 3 & 200 & 100 & 300 & 1 & 0 & 0 & 0 & 0 & 0 & 0 & 0 & 0 & 1 & 9030 \\
\hline & 4 & 300 & 0 & 300 & 1 & 0 & 0 & 0 & 0 & 0 & 0 & 0 & 0 & 1 & 9330 \\
\hline \multirow{4}{*}{ 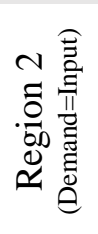 } & 5 & 0 & 523 & 523 & 0 & 1 & 0 & 1 & 0 & 0 & 1 & 0 & 0 & 0 & 21900 \\
\hline & 6 & 200 & 332 & 523 & 1 & 1 & 0 & 0 & 0 & 0 & 0 & 0 & 0 & 0 & 24930 \\
\hline & 7 & 400 & 132 & 523 & 1 & 0 & 0 & 0 & 0 & 0 & 0 & 0 & 0 & 1 & 9330 \\
\hline & 8 & 523 & 0 & 523 & 1 & 0 & 0 & 0 & 0 & 0 & 0 & 0 & 0 & 1 & 9330 \\
\hline \multirow{4}{*}{ 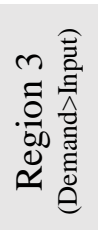 } & 9 & 0 & 700 & 700 & 0 & 1 & 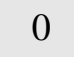 & 1 & 0 & 0 & 2 & 0 & 0 & 0 & 21900 \\
\hline & 10 & 250 & 450 & 700 & 1 & 1 & 0 & 0 & 0 & 0 & 0 & 0 & 0 & 0 & 25230 \\
\hline & 11 & 500 & 200 & 700 & 1 & 1 & 0 & 0 & 0 & 0 & 0 & 0 & 0 & 0 & 14610 \\
\hline & 12 & 700 & 0 & 700 & 1 & 0 & 0 & 0 & 0 & 0 & 0 & 0 & 0 & 1 & 9330 \\
\hline
\end{tabular}

The first case of the third region is similar to the first case of the second region where Cluster 1 products should be remanufactured in order to satisfy the demand for Cluster 2 products. Cases 10 and 12 are similar to Cases 6 and 8 respectively. However, in Case 11 although the demand for the cheaper cluster is higher, the total demand for each of the clusters are high enough such that refurbishing and selling them in their own cluster reveals the maximum profit.

Figure 6 illustrates an overview of the recovery strategies and the corresponding recovery profits under different demand scenarios. Based on the trends demonstrated in Figure 6, three observations can be done:

1- As expected, demand has a direct effect on the recovery profit. Generally, as the total demand for the recovered products increases, the profit of the recovery operations increases as well. This point can be confirmed by comparing the corresponding profits of Regions 1, 2 and 3.

2- Because of the relatively high costs of the remanufacturing and possible subsequent holding costs which may be imposed on the firm upon recovering products into one cluster more than the available demand, remanufacturing is only profitable when the demand for the more valuable cluster is high enough (i.e., Cases 5 and 9). 
3- In each region, as the demand for the more valuable cluster increases (i.e., Cluster 2 products that have higher market values) the final profit of the recovery operations increases as well. The only exception to this is Case 5 that has higher Cluster 2 demand compared to Case 6 but provides less profit. The reason is that since the firm is required to satisfy the demand for more Cluster 2 products in Case 5, remanufacturing should be done, which imposes more costs on the firm.

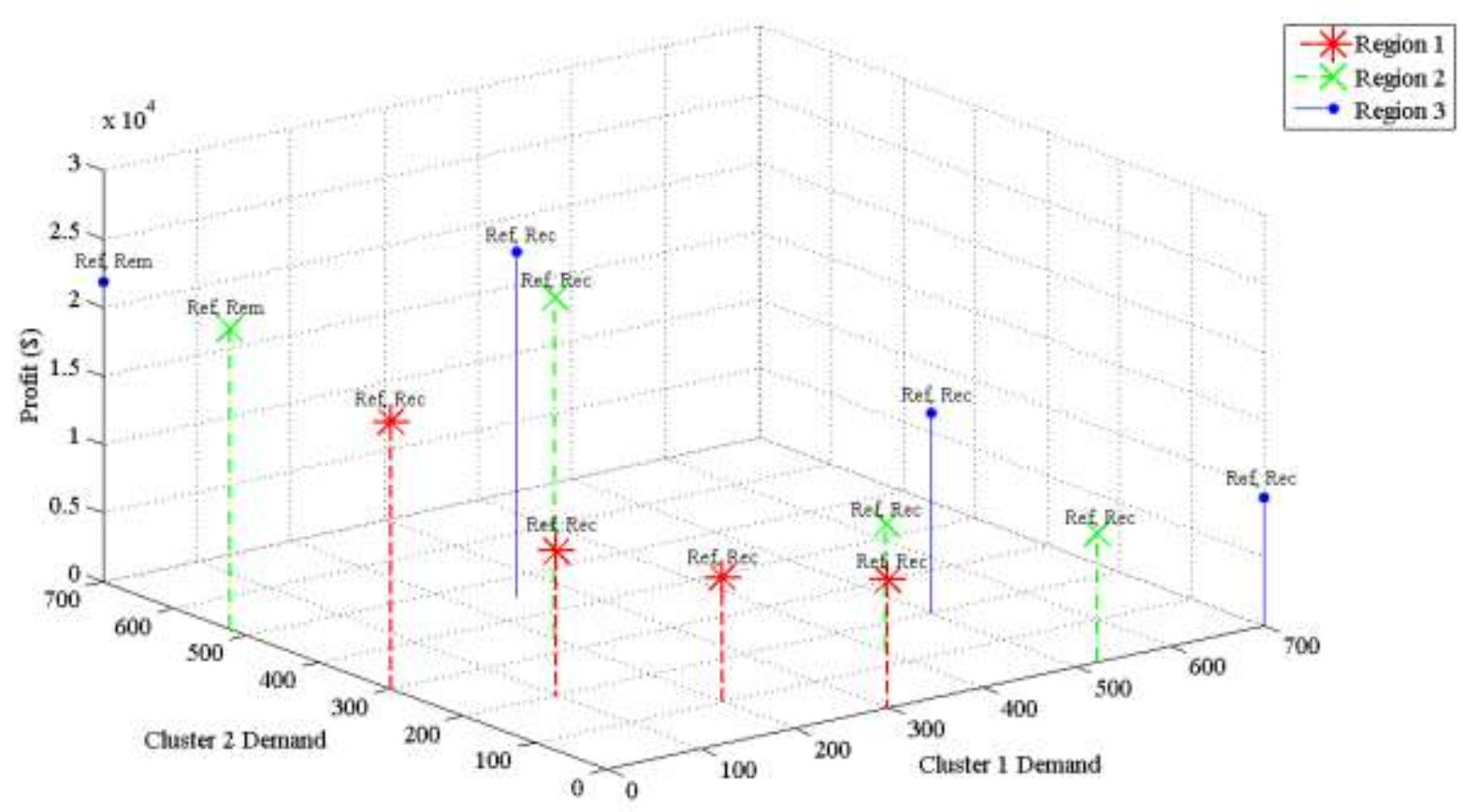

Figure 6 - Overall trend of the optimum recovery strategies and the corresponding profit under different demand assumptions. Ref $=$ Refurbish, Rem $=$ Remanufacture and Rec $=$ Recycle.

\section{6- Conclusions}

Proper value recovery of used consumer electronics has been an attraction to and yet a challenge for the industry. However, the presence of various sources of uncertainties barricades the efforts that have been made to ease the process and make it more efficient. While several methodologies have been implemented for optimizing EoL/EoU decision making, utilization of product life cycle information in the EoU decision making process seems promising. This study introduced a method in which product life cycle data are used to determine a data driven sorting policy that helps remanufacturers cluster and categorize the used incoming products and further determine the best EOU recovery option for each cluster. Resources and current sensors embedded in electronic devices like Self-Monitoring Analysis and Reporting Technology (S.M.A.R.T.) of hard drives provide available helpful information about the life cycle of the products.

A general reusability index based on the critical product features, as a measure for the product quality level, has been proposed. This measure was incorporated into the EoU decision making optimization in order to sort the items and find the best recovery strategy that maximizes the remanufacturer's profit. 
Clustering algorithms have been utilized so that similar products are identified and the same strategies are applied to them in order to decrease the dimension of the optimization problem and ease the decision making process. Clustering products and applying EOU recovery decision for each product cluster have the potential to reduce the operational costs of remanufacturing activities compared to the case that a different set of operations is considered for each individual product. A data set of computer hard drive life cycle information coupled with a set of simulated data has been utilized as a case study and a sensitivity analysis has been performed to further analyze the dependency of the results to the demand.

This work can be improved in several ways. The data available to us were limited to the hard drives data. Therefore, we had to use simulated data for other components to test the model. Incorporating other components data into the framework will enrich the model and enables us to further test the capabilities of the proposed structure in real cases. The common limitations of the clustering methodologies are also valid here. The performance of the model depends on the underlying data and their ability to be well clustered.

The performance of all recovery operations heavily depends on the demand. One of the major challenges in remanufacturing planning is the uncertain nature of the available demand. However, considering stochastic demand is beyond the scope of this study. Nonetheless, incorporating the ability to handle stochastic demand to the model will improve the reliability of the results.

\section{Acknowledgments:}

This material is based upon work supported by the National Science Foundation - USA under grant \# CMMI-1435908.

\section{References:}

[1] Esmaeilian, B., Behdad, S., and Wang, B., 2016, "The evolution and future of manufacturing: A review," J. Manuf. Syst., 39, pp. 79-100.

[2] Souza, G. C., 2013, "Closed-Loop Supply Chains: A Critical Review, and Future Research," Decis. Sci., 44(1), pp. 7-38.

[3] Fleischmann, M., Bloemhof-Ruwaard, J. M., Beullens, P., and Dekker, R., 2004, "Reverse logistics network design," Reverse Logistics, Springer, pp. 65-94.

[4] “GIVING NEW LIFE TO USED SMARTPHONES \& OTHER DEVICES” [Online]. Available: https://www.gazelle.com/. [Accessed: 12-Jul-2016].

[5] Zikopoulos, C., and Tagaras, G., 2007, "Impact of uncertainty in the quality of returns on the profitability of a single-period refurbishing operation,” Eur. J. Oper. Res., 182(1), pp. 205-225.

[6] Denizel, M., Ferguson, M., and Souza, G. G. C., 2010, "Multiperiod remanufacturing planning with uncertain quality of inputs," IEEE Trans. Eng. Manag., 57, pp. 394-404.

[7] ARAS, N., BOYACI, T., and VERTER, V., 2004, "The effect of categorizing returned products in remanufacturing," IIE Trans., 36, pp. 319-331.

[8] Ferguson, M., Guide, V. D., Koca, E., and Souza, G. C., 2009, "The value of quality grading in remanufacturing," Prod. Oper. Manag., 18(3), pp. 300-314.

[9] Robotis, A., Boyaci, T., and Verter, V., 2012, "Investing in reusability of products of uncertain remanufacturing cost: The role of inspection capabilities," Int. J. Prod. Econ., 140(1), pp. 385395. 
[10] Shin, J.-H., Kiritsis, D., and Xirouchakis, P., 2015, "Design modification supporting method based on product usage data in closed-loop PLM,” Int. J. Comput. Integr. Manuf., 28(6), pp. 551-568.

[11] Sabbaghi, M., Esmaeilian, B., Raihanian Mashhadi, A., Cade, W., and Behdad, S., 2015, "Reusability Assessment of Lithium-Ion Laptop Batteries Based on Consumers Actual Usage Behavior,” J. Mech. Des., 137(12), p. 124501.

[12] Scheidt, L., and Zong, S., 1994, "An approach to achieve reusability of electronic modules," Electron. Environ. 1994. ISEE 1994., Proceedings., 1994 IEEE Int. Symp., pp. 331-336.

[13] Nikolaidis, Y., 2009, "A modelling framework for the acquisition and remanufacturing of used products,” Int. J. Sustain. Eng., 2(3), pp. 154-170.

[14] Panagiotidou, S., Nenes, G., and Zikopoulos, C., 2013, "Optimal procurement and sampling decisions under stochastic yield of returns in reverse supply chains," OR Spectr., 35(1), pp. 1-32.

[15] Pishvaee, M. S., Jolai, F., and Razmi, J., 2009, “A stochastic optimization model for integrated forward/reverse logistics network design,” J. Manuf. Syst., 28, pp. 107-114.

[16] Fathi, M., Zandi, F., and Jouini, O., 2015, "Modeling the merging capacity for two streams of product returns in remanufacturing systems," J. Manuf. Syst., 37, Part 1, pp. 265-276.

[17] Galbreth, M. R., and Blackburn, J. D., 2010, "Optimal Acquisition Quantities in Remanufacturing with Condition Uncertainty,” Prod. Oper. Manag., 19(1), pp. 61-69.

[18] Subulan, K., Baykasoğlu, A., Özsoydan, F. B., Taşan, A. S., and Selim, H., 2015, “A case-oriented approach to a lead/acid battery closed-loop supply chain network design under risk and uncertainty," J. Manuf. Syst., 37, Part 1, pp. 340-361.

[19] Francie, K. A., Jean-Pierre, K., Pierre, D., Victor, S., and Vladimir, P., 2015, "Stochastic models and numerical solutions for manufacturing/remanufacturing systems with applications to the printer cartridge industry," J. Manuf. Syst., 37, Part 3, pp. 662-671.

[20] "Estimate Your Trade-In Value" [Online]. Available: http://www.bestbuy.com/site/misc/best-buytrade-in/pcmcat133600050011.c?id=pcmcat133600050011. [Accessed: 12-Jul-2016].

[21] Klausner, M., Grimm, W. M., Hendrickson, C., and Horvath, A., 1998, "Sensor-based data recording of use conditions for product takeback," Electron. Environ. 1998. ISEE-1998. Proc. 1998 IEEE Int. Symp., pp. 138-143.

[22] Klausner, M., Grimm, W. M., and Hendrickson, C., 1998, "Reuse of Electric Motors in Consumer Products," J. Ind. Ecol., 2(2), pp. 89-102.

[23] Simon, M., Bee, G., Moore, P., Pu, J.-S., and Xie, C., 2001, "Modelling of the life cycle of products with data acquisition features," Comput. Ind., 45(2), pp. 111-122.

[24] Teunter, R. H., and Flapper, S. D. P., 2011, "Optimal core acquisition and remanufacturing policies under uncertain core quality fractions,” Eur. J. Oper. Res., 210(2), pp. 241-248.

[25] Jin, X., Hu, S. J., Ni, J., and Xiao, G., 2013, “Assembly Strategies for Remanufacturing Systems With Variable Quality Returns,” IEEE Trans. Autom. Sci. Eng., 10(1), pp. 76-85.

[26] Ondemir, O., and Gupta, S. M., 2014, "Quality management in product recovery using the Internet of Things: An optimization approach," Comput. Ind., 65(3), pp. 491-504.

[27] Jun, H.-B., Shin, J.-H., Kim, Y.-S., Kiritsis, D., and Xirouchakis, P., 2009, “A framework for RFID applications in product lifecycle management,” Int. J. Comput. Integr. Manuf., 22(7), pp. 
$595-615$.

[28] Zikopoulos, C., and Tagaras, G., 2008, "On the attractiveness of sorting before disassembly in remanufacturing,” IIE Trans., 40(3), pp. 313-323.

[29] Van Wassenhove, L. N., and Zikopoulos, C., 2010, "On the effect of quality overestimation in remanufacturing,” Int. J. Prod. Res., 48(18), pp. 5263-5280.

[30] Parlikad, A. K., and McFarlane, D., 2010, "Value of information in product recovery decisions: A Bayesian approach,” Int. J. Sustain. Eng., 3(2), pp. 106-120.

[31] Reveliotis, S. A., 2007, "Uncertainty management in optimal disassembly planning through learning-based strategies," IIE Trans., 39(6), pp. 645-658.

[32] Hu, C., Youn, B. D., Wang, P., and Taek Yoon, J., 2012, "Ensemble of data-driven prognostic algorithms for robust prediction of remaining useful life," Reliab. Eng. Syst. Saf., 103, pp. 120 135.

[33] Wang, P., Youn, B. D., Xi, Z., and Kloess, A., 2009, "Bayesian reliability analysis with evolving, insufficient, and subjective data sets," J. Mech. Des., 131(11), p. 111008.

[34] Sabbaghi, M., Esmaeilian, B., Raihanian Mashhadi, A., Behdad, S., and Cade, W., 2015, “An investigation of used electronics return flows: A data-driven approach to capture and predict consumers storage and utilization behavior," Waste Manag., 36, pp. 305-315.

[35] Raihanian Mashhadi, A., and Behdad, S., 2016, "Integration of Product Life Cycle Data toward Remanufacturing of Waste Electrical and Electronic Equipment," IIE Annual Conference and Expo, Institute of Industrial and Systems Engineers, Anaheim, CA, USA.

[36] Beach, B., "How long do disk drives last?” [Online]. Available: https://www.backblaze.com/blog/how-long-do-disk-drives-last/. [Accessed: 01-Jan-2016].

[37] Mazhar, M. I., Kara, S., and Kaebernick, H., 2007, "Remaining life estimation of used components in consumer products: Life cycle data analysis by Weibull and artificial neural networks," J. Oper. Manag., 25(6), pp. 1184-1193.

[38] Rugrungruang, F., Kara, S., and Kaebernick, H., 2009, “An integrated methodology for assessing physical and technological life of products for reuse,” Int. J. Sustain. Manuf., 1(4), pp. 463-490.

[39] Clottey, T., and Benton, W. C., 2014, "Determining core acquisition quantities when products have long return lags," IIE Trans., 46(9), pp. 880-893.

[40] Hartigan, J. A., and Wong, M. A., 1979, “Algorithm AS 136: A k-means clustering algorithm,” J. R. Stat. Soc. Ser. C (Applied Stat., 28(1), pp. 100-108.

[41] Rousseeuw, P. J., 1987, "Silhouettes: A graphical aid to the interpretation and validation of cluster analysis," J. Comput. Appl. Math., 20, pp. 53-65.

[42] Tibshirani, R., Walther, G., and Hastie, T., 2001, "Estimating the number of clusters in a data set via the gap statistic,” J. R. Stat. Soc. Ser. B (Statistical Methodol., 63(2), pp. 411-423. 